\title{
Subsurface Analysis of Oil Paintings by Means of Scanning Macro-XRF
}

\author{
K. Janssens, ${ }^{*}$ M. Alfeld, * J. Dik, ** and K. Rickers *** \\ * Department of Chemistry, University of Antwerp, Universiteitsplein 1, B-2610 Antwerp, Belgium \\ ** Department of Engineering, Delft University of Technology, 2628 CN Delft, The Netherlands \\ *** Hamburg Synchrotron Laboratory, DESY, Notkestrasse 85, D-22607 Hamburg,Germany
}

From the point of view of a material scientist, easel paintings can be considered as extended and complex multilayered objects, where a variety of materials of different density and physico-chemical properties is applied on top of each other in various layers. When a painting contains only one pictorial (i.e., visual information carrying) layer, the number of strata can be limited to three or four, i.e., (a) the substrate material (e.g., canvas, wood or metal), (b) a ground layer (e.g., a glue-chalk mixture or a glue-gypsum base), (c) the actual pictorial layer and (d) an optional varnish layer. In many paintings, the pictorial layer itself is composed of more than one superimposed level in order to achieve an particular optical effect or because the artists altered or corrected his own design while first creating the work. Quite a few paintings also contain more than one pictorial layer; this is the case for paintings that, immediately upon their creation or several years/decades afterwards, were painted over to re-use the canvas or panel. Usually it concerns paintings from the early (or earlier) period of a painter with which the artist grew dissatisfied in later stages of his career so that a new set of ground+pictorial layers were used to cover up the layers already present. For these reasons, many contemporary art-historians, on the other hand, are interested to obtain information on the covered-up paintings.

At a given location on the painting and provided the layers are not too thin (i.e., above 5-10 $\mu \mathrm{m}$ ), such a complex multilayer system can be non-destructively analyzed in terms of the elemental content of the individual layers via depth profiling by means of confocal $\mu$-XRF [1]. While in principle, also the imaging of the distribution of the elemental contents in each layer separately might be possible by the same technique, in practice, this would be a quite challenging and time consuming exercise because of the irregularity of the absolute depth and thickness of the layers relative to the surface and relative to the coordinate system of the confocal analysis system. Therefore, rather than employ a full 3D-selective manner of imaging, it was decided to employ the more conventional, two-dimensional projective approach of 'regular' $\mu$-XRF for imaging of (superficial and buried) layers in oil paintings. In order to exploit the benefits of this approach to the fullest while at the same time minimizing the danger of inflicting damage to valuable works of art, several choices needed to be made regarding the manner in which the painting was irradiated (X-ray source, exposure time, primary energy, beam size) and the manner in which the fluorescent radiation was detected (number and type of detectors, position(s) relative to painting and primary beam).

We have been able to demonstrate that a proper selection of the above mentioned experimental parameters permits, in a completely non-destructive manner, to obtain information about buried layers of the pictorial composition that previously were not visible to the naked eye or to conventional imaging methods such as X-ray radiography or infra-red reflectography [2]. Vincent Van Goghs 'Patch of Grass' and other paintings were transported to the microfluorescence beamline L at DORIS-III, a second-generation synchrotron light source at HASYLAB (Hamburger Synchrotronstrahlungslabor at Deutsches Elektronen-Synchrotron DESY, Hamburg, Germany). In 
the X-ray radiographs of this canvas, the outline of a person's head is (just) visible. Elemental distribution images were obtained by scanning an area of $15 \times 15 \mathrm{~cm}^{2}$ by means of a $0.5 \times 0.5 \mathrm{~mm}^{2}$ primary X-ray beam of $30 \mathrm{keV}$ energy. These maps could be compared with features of the surface painting as well as the X-ray radiographic image. Most elemental distributions reflect the variation in pigment and colour in the top layer of 'Patch of Grass', including various transition metals such as $\mathrm{Mn}, \mathrm{Co}, \mathrm{Fe}$ and $\mathrm{Cu}$. The maps of two elements are directly related to the covered head: $\mathrm{Hg}$ and $\mathrm{Sb}$, while some features in the Zn-map are also related to it. Mercury can be associated with vermillion, i.e., the red pigment mercury sulfide. As expected, this pigment was found predominantly in the lips and cheek. The Sb distribution shows a very clear overlap with the lighted parts of the head, but its chemical origin was not immediately obvious. In order to identify the chemical Sb-compound, Xray absorption near-edge spectroscopy (XANES) measurements were performed on the painting at the X-ray absorption spectroscopy beamline $\mathrm{C}$ at HASYLAB at selected positions on the head. The resulting data indicate that $\mathrm{Sb}_{2} \mathrm{O}_{3}$, a white pigment introduced in the 1920s, can be excluded as possible source. The XANES spectra are rather similar to that of lead antimonate yellow, a pigment commonly used by Van Gogh. We conclude that the distribution of $\mathrm{Hg}$ corresponds to the red component of the pinkish flesh tones, while $\mathrm{Sb}$ relates to a yellowish white. A tri-tonal reconstruction, in which the maps for $\mathrm{Hg}$ and $\mathrm{Sb}$ have been colorized, was made, presenting a significantly clearer and more detailed image of the hidden composition.

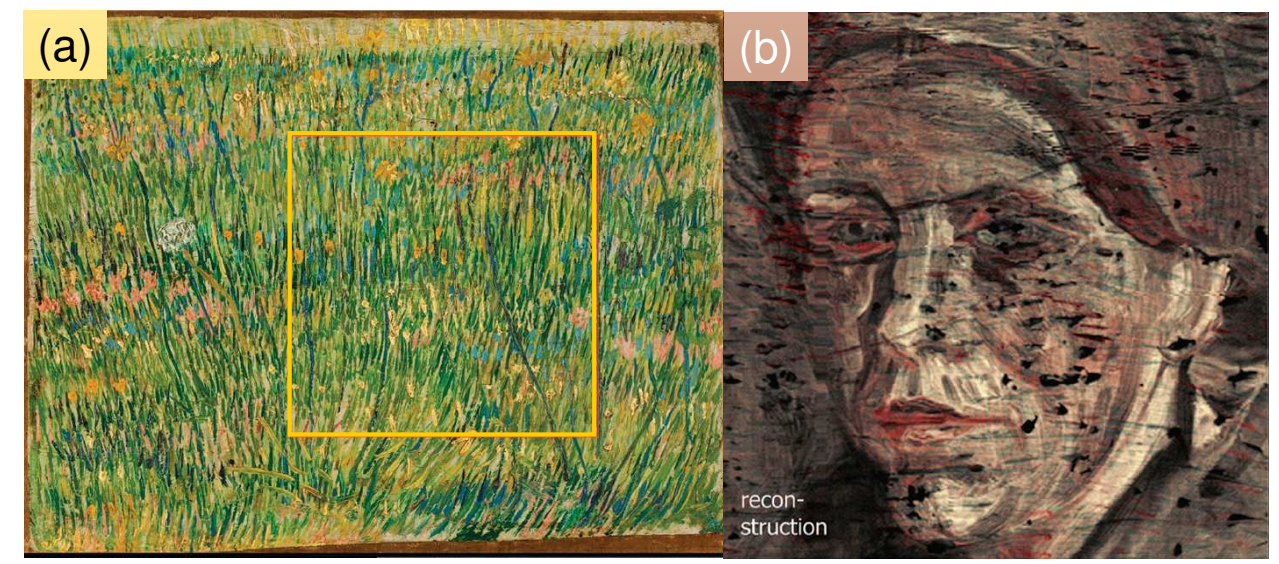

FIG. 1. (a) Photograph of 'Patch of Grass', V. Van Gogh; (b) tri-tonal reconstruction (from [2]).

We are currently exploring the possibilities of employing laboratory scanning macro-XRF equipment for accomplishing similar results as previously obtained at SR facilities. The approach we have taken here is to construct a macro-XRF spectrometer capable of performing a scanning movement in front of a stationary painting so that it can operate in an unperturbed manner in many galleries or museum depots with minimal ancillary equipment and/or facilities and with minimal risks to the works of art [3]. In order to reduce the total scanning time, a system incorporating multiple compact XRF detectors is now in use to study, e.g., underpaintings in $17^{\text {th }}$. C. paintings.

References

[1] Z. Smit, K. Janssens, K. Proost et al., Nucl. Instr. Phys. Res. B 219 (2004) 35-40.

[2] J. Dik, K. Janssens, G. Van Der Snickt et al., Anal. Chem. 80 (2008) 6436-6442.

[3] G. Vittiglio, K. Janssens, B. Vekemans et al., Spectrochimica Acta B 54 (1999) 1697-1710.

[4] This research was supported by Belgian Science Policy IUAP VI/16 and NWO VENI grants. 